\title{
Association between metalloproteinases 2 and 9 activity and ERK1/2 phosphorylation status in head and neck cancers: An ex vivo study
}

\author{
WERNER GARAVELLO $^{1,2}$, DANIELE MAGGIONI ${ }^{1}$, GABRIELLA NICOLINI $^{1}$, \\ LORENZO MOTTA $^{2}$, GIOVANNI TREDICI ${ }^{1}$ and RENATO GAINI ${ }^{2}$ \\ ${ }^{1}$ Department of Neuroscience and Biomedical Techologies, University of Milan-Bicocca and \\ ${ }^{2}$ Department of Otorhinolaryngology, Head and Neck Surgery, San Gerardo Hospital, \\ University of Milan-Bicocca, Via Cadore 48, I-20052 Monza, Italy
}

Received May 11, 2010; Accepted June 18, 2010

DOI: 10.3892/or_00000957

\begin{abstract}
Advances in clinical treatment of head and neck squamous cell carcinoma (HNSCC) are hampered by its high infiltrative potential leading to distal metastasis. Since their ability to degrade the basal lamina and extracellular matrix, matrix metalloproteinases (MMP) have a pivotal role in tumor invasion. The overexpression and the aberrant activity of MMPs especially of MMP2 and MMP9, during HNSCC development and progression have been reported. However, up to now little is known about the mechanism of their regulation in HNSCC. It has been demonstrated that MMP2/9 expression is negative regulated by extracellular signal regulated kinase 1 and $2($ ERK1/2) in HNSCC cell lines. ERKs are protein kinases belonging to the mitogenactivated protein kinases family, and they are involved in the regulation of different cellular aspects, from apoptosis to cell proliferation and differentiation. In the present study we evaluated MMP2 and MMP9 activity by gelatine zymography in 16 tissue samples of HNSCC and their paired normal mucosa from patients undergoing surgical treatment. Moreover, ERK1/2 activation was analyzed by immunoblotting. A statistically significant decrease in the levels of activated ERK2 in cancer specimens in comparison with paired normal tissues was observed, whereas a significant increase in the activity of MMP2 was found in cancer specimens. However, the statistical analysis failed to demonstrate a correlation between the increase in MMP2 activity and the reduction of ERK $1 / 2$ activation levels. The results obtained, therefore, rule out, for the first time in an
\end{abstract}

Correspondence to: Dr Daniele Maggioni, Department of Neuroscience and Biomedical Techologies, University of Milan-Bicocca, Via Cadore 48, I-20052 Monza, Italy

E-mail: daniele.maggioni1@unimib.it

Key words: head and neck squamous cell carcinoma, ERK, metalloproteinase 2, metalloproteinase 9 ex vivo study, the existence of a negative correlation between ERK1/2 activation and MMP2 activity.

\section{Introduction}

Head and neck squamous cell carcinoma (HNSCC) is the sixth most frequent tumor in the world, affecting almost 1 million people per year worldwide, with a steady increase in incidence in developing countries (1). The majority of HNSCCs arises from oral cavity and larynx epithelia. In the American population the former shows an incidence of 35750 cases, the latter has an incidence of 12290 cases per year (1).

Therapy relies on surgery, radiotherapy, chemotherapy, and recently, on the use of molecularly targeted agents (e.g., EGFR), however, it is hampered by the high infiltrative potential, resulting in loco-regional and distant metastasis spreading, which reduce the 5-year cause-specific survival rate of about $50 \%$ (2).

Tumor spreading, which refers to the migration of cancer cells through extracellular stroma, depends on the degradation of the basement membrane (BM) and extracellular matrix (ECM) surrounding the tumor. ECM may be degradated by a variety of proteolytic enzymes, with matrix metalloproteinases (MMPs) having the pivotal role. MMPs are a family of zinc-dependent endopeptidases that comprises a large number of proteinases. Among them, MMP2 and MMP9, namely gelatinase A and B, play a key role in tumor invasion (3), since they are able to digest collagen type IV, which is the main component of BM (4). Enhanced expression and activity of MMP2 and MMP9 have been shown in different kinds of cancers (5), including HNSCC $(6,7)$. However, the mechanisms underlying their up-regulation remain largely unknown. MMP2/9 activity, may be regulated at different levels, both at the trascriptional and at the activation level (8). MMP2/9 are transcriptionally induced by growth factors and cytokines through the nuclear factor- $\mathrm{KB}$ and AP-1 transcription factors and, in addition, their activity in tissues is regulated by specific inhibitors, TIMPs (tissue inhibitor of metalloproteinase) (9). 
Substantial evidence has highlighted the involvement of mitogen-activated protein kinases (MAPKs), and especially extracellular signal-regulated kinases 1 and 2 (ERK1/2) in the regulation of MMP expression and activity $(10,11)$. However, data are controversial since MAPK pathways cross-talk and cell type specific responses make it difficult to state clearly the relationship between MAPK and MMP2/9 activity.

MAPKs are a family of serine/threonine kinases that are important in converting extracellular stimuli into a wide range of cellular responses, including proliferation, differentiation and apoptosis $(12,13)$. Three subfamilies of MAPK have been identified and described in detail: ERK, JNK/ SAPK (c-Jun N-terminal kinase/stress-activated protein kinase) and p38. Each MAPK pathway presents an activation module that relies on the serial phosphorylations on tyrosin and threonine residues, whereas MAPKs are switched off by dephosphorylation by a specific phosphatase (14).

ERK1 and ERK2, also referred to as p44 and p42, belong to the well described oncogenic pathway that comprises the proto-oncoproteins Ras and Raf, which are the main upstream activators of ERK1/2 (12). Extracellular signals such as cytokines and growth factors trigger ERK1/2 activation. After activation ERK1/2 can phosphorylate and activate different downstream targets, either citoplasmatic or nuclear, including cytoskeletal proteins and transcription factors such as AP-1, c-Fos and Elk-1 (15). According to this complex role in determining either proliferation or cell death, aberrant activation of ERK pathway is frequently seen in cancers (16-18).

A correlation between ERK1/2 phosphorylation status and gelatinase activity in oral SCC has been hypothesized (19). Thus, in the present study we evaluated whether a correlation would exist, analysing ERK1/2 activation status and gelatinase activity in cancer specimens in comparison to adjacent normal mucosa by immunoblotting and gelatine zymography, respectively. The potential association between gelatinase activity and ERK1/2 phosphorylation was also evaluated.

\section{Materials and methods}

Materials for this study were specimens of HNSCC, histologically confirmed by a previous biopsy, and adjacent normal mucosa from 16 patients, who underwent surgical resection at the Department of Otorhinolaryngology of the San Gerardo Hospital, Monza, Italy, between March 1, 2007 and April 30, 2009.

The mean patient age was $63.8 \pm 10.8$ years (range 42 84 years), none had evidence either of neck nodes nor distant metastases at the time of admission. All the tumor samples were histologically classified as squamous cell carcinomas. According with the number of mitosis within cancer specimens and with the degree of differentiation of cancer cells when compared with their normal counterparts, carcinomas were classified by grading from 1 to 3 : G1, welldifferentiated; G2, moderately differentiated; and G3, poorly differentiated. Two biopsy specimens were graded 1 (G1), 8 were G2 and 6 were G3. All the patients reported alcohol ( $>1$ litre/day) and tobacco ( $>20$ cigarettes/day) consumption.
A tumor sample for each case was collected, avoiding the eventual necrotic center, as well a control sample corresponding to a specimen of mucosa taken from a site adjacent to the resection margins, free from neoplastic infiltration as confirmed by a subsequent histological examination.

Tissue samples were dissected and divided into three specimens, the first for histological evaluation, the second for gelatine zymography analysis and the third for the immunoblot analysis. Samples for immunoblotting and zymography were immediately frozen in liquid nitrogen and stored at $-70^{\circ} \mathrm{C}$. Patients enrolled in this study have been informed of the purposes of the study and gave written consent.

Western blotting. Tissues for SDS-PAGE were homogenized on ice in lysis buffer (10\% glycerol, $25 \mathrm{mM}$ Tris- $\mathrm{HCl} \mathrm{pH} \mathrm{7.5,}$ $1 \%$ Triton X-100, 5 mM EDTA, 1 mM EGTA) containing freshly added protease and phosphatase inhibitors (4 mM phenylmethylsulfonyl fluoride, $1 \%$ aprotinin, $10 \mathrm{mM}$ sodium orthovanadate, $20 \mathrm{mM}$ sodium pyrophosphate). Protein content in the extract was measured by the Bradford assay. Protein lysates $(60 \mu \mathrm{g})$ were resolved on a $13 \%$ polyacrylamide gel and then transferred onto a nitrocellulose filter (Amersham, Cologno Monzese, Monza, Italy). After brief washing, membranes were blocked in TBST [10 mM Tris- $\mathrm{HCl}(\mathrm{pH} 7.5)$, $150 \mathrm{mM} \mathrm{NaCl}$ and $0.1 \%$ Tween-20] containing 5\% non-fat milk for $1 \mathrm{~h}$ at room temperature.

Membranes were subsequently incubated overnight at $4^{\circ} \mathrm{C}$ in $5 \%$ BSA in TBST containing primary polyclonal antibodies either anti-phospho-ERK1/2p42/44 (pERK1/2) or antipanERK1/2 ${ }^{\mathrm{p} 42 / 44}$ (both from Cell Signalling, Beverly, MA) or anti-actin (Santa Cruz Biotechnology, Santa Cruz, CA), followed by a secondary antibody (horseradish peroxidaseconjugated anti-IgG; Perkin-Elmer, Monza, Italy). Finally, the immune complexes were visualized using the ECL chemiluminescence system (Genespin, Milan, Italy), digitized and quantified by densitometric analysis of non-saturated autoluminographs using the Gel Logic 100 Imagine System (Eastman Kodak Co., Rochester, NY). Actin immunoblot analysis was used as a loading control.

Results are expressed as a relative expression (RE) considering the ratio between the optical density of the under study protein band and the one of the corresponding actin band. Finally, the levels of expression of each protein in normal tissue were set to 1 . The relative expression of the protein under study in each tumor sample was obtained comparing the value in the tumor sample with its correspondent normal tissue.

Gelatin zymography. Specimens for gelatine zymography analysis were lysed in $10 \mathrm{mM}$ Tris- $\mathrm{HCl}(\mathrm{pH} 7.5)$ and $0.25 \%$ Triton X-100. Protein concentration was determined using the Bradford method. Protein lysates were subsequently subjected to electrophoresis on a $10 \%$ SDS-PAGE gel containing $2 \mathrm{mg} / \mathrm{ml}$ gelatin from porcine skin (Sigma-Aldrich, St. Louis, MO). The resulting gels were subsequently washed twice for 15 min in Triton X-100 2.5\% to remove the SDS and finally incubated for $48 \mathrm{~h}$ in activation buffer containing $150 \mathrm{mM} \mathrm{NaCl}, 50 \mathrm{mM}$ Tris- $\mathrm{HCl} \mathrm{pH} 7.5$ and $5 \mathrm{mM} \mathrm{CaCl}_{2}$.

After the incubation gel was stained for $2 \mathrm{~h}$ with $0.25 \%$ Comassie Brilliant Blue R250 (Sigma-Aldrich) in 45\% 
$\mathbf{A}$

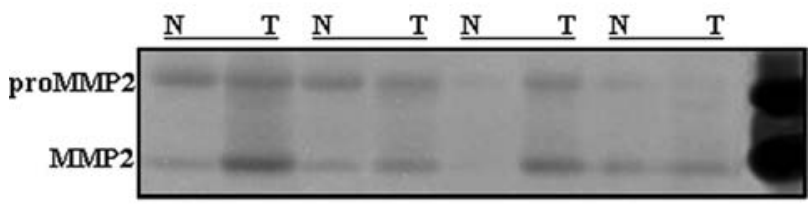

B

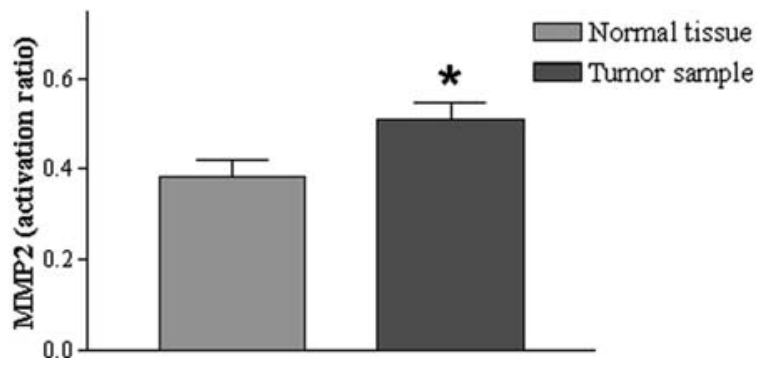

Figure 1. MMP2 activity was increased in cancer specimens when compared with normal tissues. (A) Representative image showing MMP2 activity in four pairs of HNSCC specimens $(\mathrm{T})$ and surrounding normal mucosa $(\mathrm{N})$; the purified MMP2 protein was loaded in the last lane. The original image has been digitally inverted. (B) MMP2 activation ratio, i.e., MMP2/ (MMP2+proMMP2), was statistically significantly increased $\left({ }^{*} \mathrm{p}<0.05\right)$ in cancer specimens in comparison with paired normal tissues (mean $\pm \mathrm{SD}$ ).

methanol and $10 \%$ acetic acid and finally destained for $1 \mathrm{~h}$ in $10 \%$ acetic acid. To confirm metalloproteinase gelatine digestion, the purified MMP2 protein (Millipore, Billerica, MA) was loaded on gel as a positive control.

The gels were digitalized using the Gel Logic 100 Imagine System (Eastman Kodak Co.) and, after digital inversion of the images, the enzymatic activity was quantified. Briefly, lysis bands were identify and the optical density calculated by the software. MMP2 activity was expressed as the activation ratio, i.e., the ratio between the active $62 \mathrm{kDa}$ form and the active form plus the inactive $72 \mathrm{kDa}$ form (proMMP2). MMP9 activity was expressed as the total activity normalized for the activity of the MMP9 standard.

Statistics. The differences in ERK1/2 expression/activation and MMP2/9 activity between cancer specimens and adjacent normal mucosa were analyzed using the paired Student's ttest. A p-value $<0.05$ was considered statistically significant. The relationship between levels of ERK1/2 activation and MMP2/9 activity were evaluated using the Pearson rank correlation analysis, with significance set at $\mathrm{p}<0.05$.

\section{Results}

Gelatin zymography analysis of MMP2 and MMP9 activity. As elevated expression of MMP2/9 has been correlated with an increased metastatic potential in many cancer types. We examined the gelatinolytic activities of MMP2 and MMP9 by gelatin zymography in $n=16 \mathrm{HNSCC}$ specimens and in their paired adjacent normal mucosa.

Fig. 1 shows a representative MMP2 zymogram of HNSCC and adjacent normal tissue samples. Densitometric
A
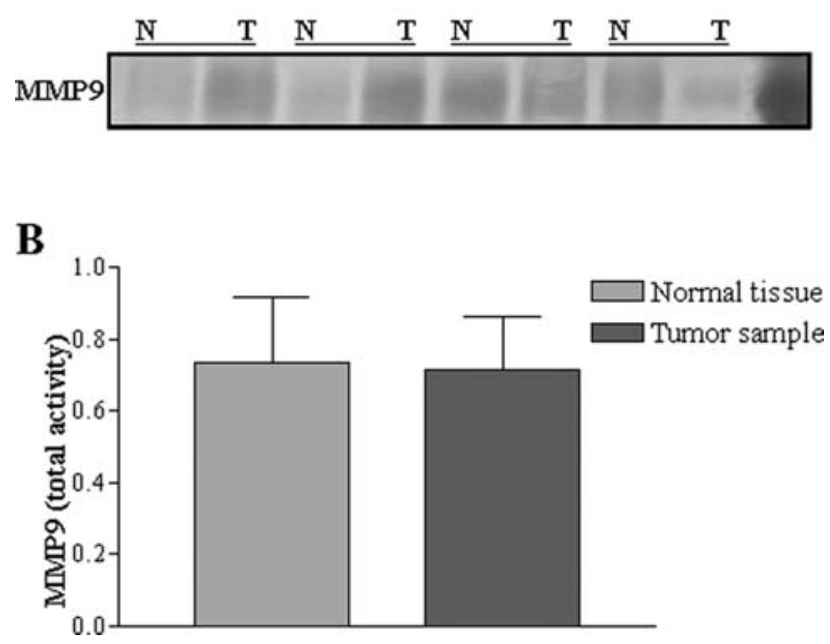

Figure 2. MMP9 activity in cancer specimens and in paired normal tissue. (A) Representative image showing MMP9 activity in four pairs of HNSCC specimens $(\mathrm{T})$ and surrounding normal mucosa $(\mathrm{N})$, the purified MMP9 protein was loaded in the last lane; the original image has been digitally inverted. (B) No differences in MMP9 activity were observed between cancer specimens and their paired surrounding normal mucosa (mean $\pm \mathrm{SD})$.

computer-assisted analyses demonstrated that the activation ratio, i.e., the ratio between the active MMP2 and the active MMP2 plus the proMMP2, was statistically significant higher $(\mathrm{p}<0.05)$ in cancer samples $(0.511 \pm 0.157$; mean $\pm S D)$ than in normal tissues $(0.373 \pm 0.142$; mean \pm SD), (Fig. 1$)$.

On the contrary, no significant difference in levels of gelatinolytic activity has been observed for MMP9. In fact, the average $( \pm$ SD) MMP9 activity was $0.626 \pm 0.277$ and $0.635 \pm 0.653$ in control tissues and in cancer specimens, respectively (Fig. 2).

Immunoblot analysis of ERK1 and ERK2 expression and activation. In this study the level of expression and activation of ERK $1 / 2$ in $n=16$ samples, obtained from surgical resection of HNSCC neoplasia, were compared with the paired adjacent normal mucosa. Using immunoblot analysis the total protein levels of ERK1/2 $2^{\mathrm{p} 4 / \mathrm{p} 42}$ as well as the activated (phosphorylated) form levels were evaluated.

Analysing the level of total ERK1/2 expression no significant difference between tumor samples and adjacent normal mucosa was observed. On the contrary the levels of pERK2 were significantly reduced in tumor samples in comparison with paired normal mucosa (Fig. 3). In fact the average $( \pm$ SD) expression of pERK2 was $1.207 \pm 0.913$ and $1.588 \pm 1.339$ in cancer and in control tissues respectively $(\mathrm{p}<0.05)$. A similar trend toward a reduction in the levels of phosphorylated form in cancer specimens was observed also for ERK1, but in this case results were not statistically significant. The graph in Fig. 3C shows the ratio between pERK1/2 levels in tumors and in control tissues. The modulation of both isoforms seems closely related and only in few samples an inverse trend of ERK1 and ERK2 was noted. The majority of patients shows a sharp reduction in the levels of pERK1/2, while only in 4 

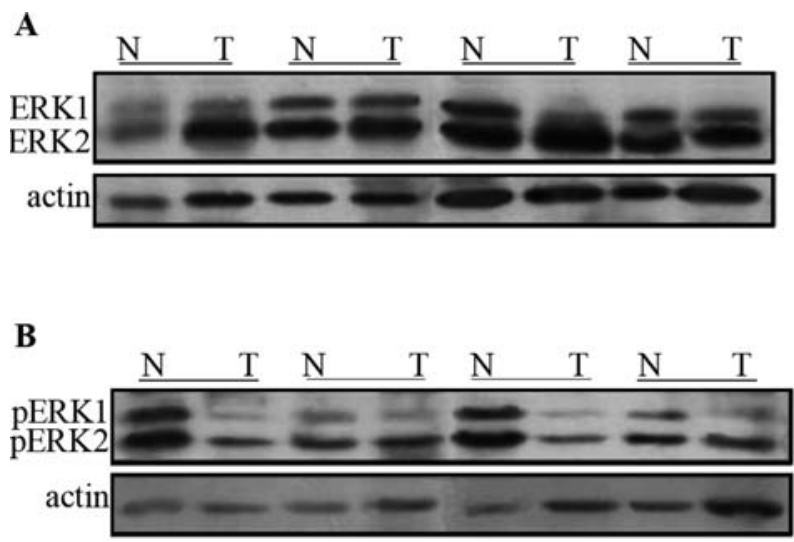

\section{C}

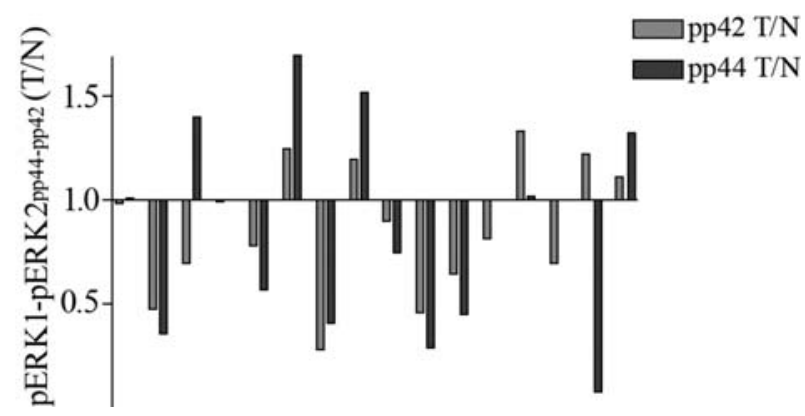

Figure 3. PhosphoERK2 levels were significantly lower in cancer specimens when compared with paired normal mucosa. (A) Representative immunoblot analysis of the levels of ERK1/2 in cancer specimens (T) and paired normal mucosa (N). (B) Representative immunoblot analysis of the levels of pERK1/2 in cancer specimens (T) and paired normal mucosa (N). (C) pERK1/2 levels have been evaluated by a densitometric analysis of autoluminographs. To control for differences in protein loading the expression of ERK1/2 was normalized to actin expression in each sample. The levels of phosphoERK2 (pp42) were statistically significantly lower in cancer specimens than in normal mucosa $(\mathrm{p}<0.05$, Student's t-test).

patients there was an increase in the levels of both isoforms in cancer.

In order to evaluate the potential correlation between the decrease in levels of ERK1/2 activation and the increased MMP2 activity observed in cancer specimens we performed a statistical regression analysis. Results did not highlight any significant correlation between the two variables under examination although a trend toward an indirect correlation between the levels of pERK1/2 and MMP2/9 activity was observed. As shown in Fig. 4, the linear regression plot between the expression levels of pERK1 and MMP2 activity in tumor and in control specimens had a Pearson's correlation coefficient of $\mathrm{R}^{2}=0.118(\mathrm{p}=0.30)$. The linear regression plot between the expression level of pERK2 and MMP2 activity in tumor and in control specimens had a Pearson's correlation coefficient of $\mathrm{R}^{2}=0.044(\mathrm{p}=0.47)$.

\section{Discussion}

The aim of this study was to investigate the potential correlation between ERK1/2 activation and MMP2/9 activity in HNSCC. Therefore, ERK1/2 phosphorylation (activation) status and MMP2/9 activity were evaluated in cancer

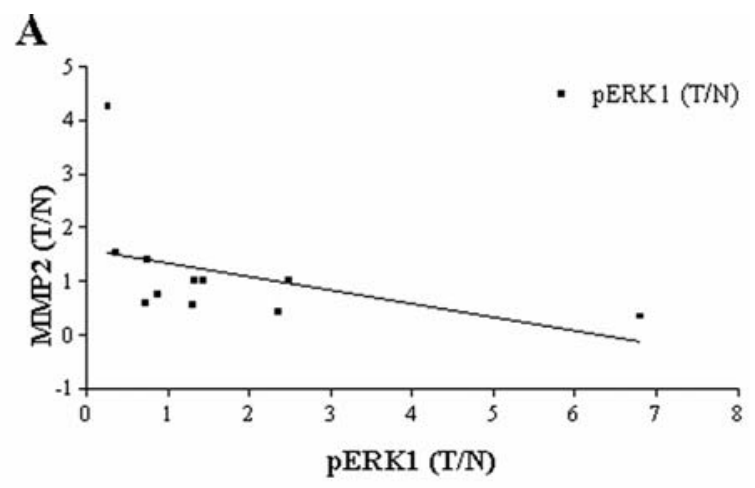

B

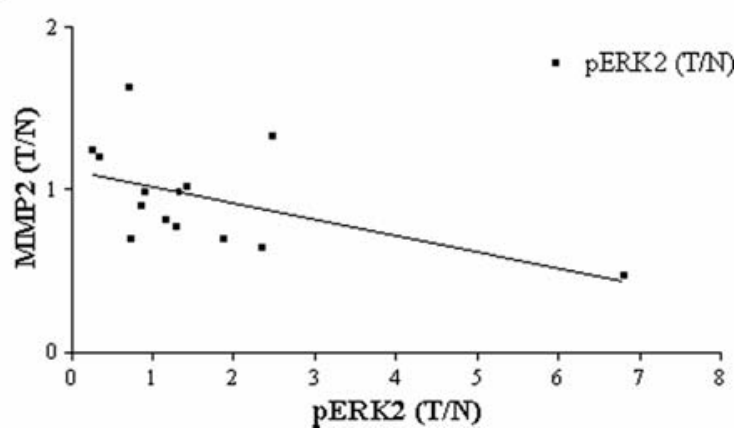

Figure 4. Scatter plots of the ratio between MMP2 activity in cancer specimens $(\mathrm{T})$ and in adjacent normal mucosa $(\mathrm{N}) \mathrm{vs}$. (A), the ratio of pERK1 levels in cancer specimens (T) and in paired normal mucosa $(\mathrm{N})$ and $(\mathrm{B})$, the ratio of pERK2 levels in cancer specimens $(\mathrm{T})$ and in paired normal mucosa (N). Linear regression analysis showed a trend toward an inverse correlation between pERK1/2 levels and MMP2 activity, with a Pearson's correlation of $\mathrm{R}^{2}=0.118$ and 0.044 , respectively.

specimens in comparison with their adjacent normal mucosa by immunoblotting and gelatine zymography, respectively.

The results obtained highlighted a deregulation of ERK1/2 activation and an increased MMP2 activity in cancer specimens, but failed to demonstrate a correlation between these two factors.

One of the main problem hampering the effectiveness of clinical strategy in HNSCC is loco-regional and distant metastasis spreading (20). Proteolytic enzymes, such as MMPs are critical for local tumor cell invasion and distant metastasis formation. In particular MMP2 and MMP9, involved in degradation of collagen type IV, the main component of basal lamina, play a pivotal role in permitting cancer cells to migrate through basal lamina and thus invading surrounding tissue (21). Many studies have underlined the role of MMPs in HNSCC invasion and lymph node metastasis $(6,8,22)$. Some of these studies, however, evaluated MMP mRNA expression or immunohistochemical positivity in the tissue, but these data may not correspond to the in vivo enzimatyc activity. Since these enzymes are secreted as zymogens and they are extracellular activated by a proteolytic cleavage, their tissue expression may not give a clear information on their activity. Therefore, in the present study, we have used gelatine zymography, a cost effective method, to analyse MMP activity in tissue samples. 
When we analysed the gelatinase activity in cancer specimens in comparison with their adjacent normal mucosa, a marked increase in the activation ratio, i.e., the ratio between the active form and the total, was found for MMP2 in cancer specimens, while a less sharp modulation was noted for MMP9. These data are consistent with studies reporting an increased MMP2 activity in HNSCC tissue $(7,23)$.

Scarce data are available regarding the regulation of MMP2 and MMP9 activity in HNSCC, however, studies carried out in oral SCC cell lines have demonstrated the ability of ERK1/2 to induce the expression of MMP2 and especially of MMP9 through activation of NF-кB $(9,24)$. A role for ERK in inducing MMP2/9 expression has been reported in several cancer cell lines (24), Munshi and colleagues (19) have raised an interesting hypothesis regarding a possible negative role of ERK $1 / 2$ in the regulation of MMP2 activity in OSCC. In fact, in OSCC cell lines they demonstrated that MMP2 activity is induced by TGF- $\beta$ through the negative mediation of ERK $1 / 2$ pathway, since ERK1/2 activation induced an increase in the levels of the MMP2 inhibitor TIMP2. These findings are supported by the observation that the TIMP2 promoter has binding sites for AP-1, one of the transcription factors activated by the ERK pathway (25). In addition, this hypothesis is consistent with the different mechanism of regulation of MMP2 and MMP9. Even though MMP2 and MMP9 share structural and functional similarities, their expression is differently regulated, while MMP9 is inducible by hormones, cytokines and growth factors through activation of different intra-cellular signalling pathways such as NF-кB and MAPK $(23,24)$, MMP2 is constitutively expressed in almost all tissues and its activity is regulated mainly at the activation level (9).

The ERKs pathway regulates many different cellular functions from cell differentiation to proliferation and apoptosis (26). Their aberrant expression and activation has been reported in several cancers, such as breast and colorectal cancer $(27,28)$.

An immunoblot analysis confirmed the deregulation of the level of the activated forms of ERK1/2 in NHSCC samples, in particular a significant decrease in the levels of phosphorylated ERK1 and ERK2 in cancer tissue when compared with their normal counterparts was highlighted. Based on these observations, we evaluated whether a relationship between ERK activation and MMP2 activity existed.

The Pearson test, did not show any statistically significant correlation between ERK1/2 activation levels and MMP2 or MMP9 activity in our specimens. Results of this study failed to support the hypothesis proposed by Munshi et al, thus indicating that the increased MMP2 activity observed in HNSCC specimens is not correlated with the decrease in the level of ERK1/2 activation. However, due to limitation in the specimens size, only a limited groups of samples were evaluated for both ERK1/2 phosphorylation and MMP2/9 activity. Therefore, further studies on a larger patients group should be carried out. In addition, since MMP regulation is strictly cell type-dependent it should be taken into consideration that the correlation between ERK activation and MMP2 activity observed in cell lines in vitro may not be confirmed in tissue samples, due to a higher cell type heterogeneity.

\section{References}

1. Jemal A, Siegel R, Ward E, Hao Y, Xu J and Thun MJ: Cancer statistics, 2009. CA Cancer J Clin 59: 225-249, 2009.

2. Rogers SJ, Harrington KJ, Rhys-Evans P, O-Charoenrat P and Eccles SA: Biological significance of c-erb family oncogenes in head and neck. Cancer Metastasis Rev 24: 47-69, 2005.

3. Bogusiewicz M, Stryjecka-Zimmer M, Szymanski M, Rechberger T and Golabek W: Activity of matrix metalloproteinases-2 and -9 in advanced laryngeal cancer. Otolaryngol Head Neck Surg 128: 132-136, 2003.

4. Gelse K, Pöschl E and Aigner T: Collagens-structure, function and biosynthesis. Adv Drug Deliv 55: 1531-1546, 2003.

5. Coussens LM, Tinkle CL, Hanahan D and Werb Z: MMP-9 supplied by bone marrow-derived cells contributes to skin carcinogenesis. Cell 103: 481-490, 2000.

6. Franchi A, Santucci M, Masini E, et al: Expression of matrix metalloproteinase 1 , matrix metalloproteinase 2 and matrix metalloproteinase 9 in carcinoma of the head and neck. Cancer 95: 1902-1910, 2002.

7. Patel V, Ramesh A, Traicoff JL, Baibakov G, Emmert-Buck MR, Gutkind JS and Knezevic V: Profiling EGFR activity in head and neck squamous cell carcinoma by using a novel layered membrane Western blot technology. Oral Oncol 41: 503-508, 2005.

8. Christopoulos TA, Papageorgakopoulou N, Ravazoula P, Mastronikolis NS, Papadas TA, Theocharis DA and Wynios DH: Expression of metalloproteinases and their tissue inhibitors in squamous cell laryngeal carcinoma. Oncol Rep 18: 855-860, 2007.

9. Zhang S, Li Z, Wu X, Huang Q, Shen HM and Ong CN: Methyl3-indolylacetate inhibits cancer cell invasion by targeting the MEK1/2-ERK1/2 signaling pathway. Mol Cancer Ther 5: 3285-3293, 2006.

10. Mendes O, Kim HT, Lungu G and Stoica G: MMP2 role in breast cancer brain metastasis development and its regulation by TIMP2 and ERK1/2. Clin Exp Metastasis 24: 341-351, 2007.

11. Chang YM, Shih YT, Chen YS, Liu CL, Fang WK, Tsai CH, Tsai FJ, Kuo WW, Lai TY and Huang CY: Schwann cell migration induced by earthworm extract via activation of PAs and MMP2/9 mediated through ERK1/2 and p38. Evid Based Complement Alternat Med, March 20, 2010 (Epub ahead of print).

12. Mebratu Y and Tesfaigzi Y: How ERK1/2 activation controls cell proliferation and cell death: is subcellular localization the answer? Cell Cycle 8: 1168-1175, 2009.

13. Scuteri A, Galimberti A, Maggioni D, Ravasi M, Pasini S, Nicolini G, Bossi M, Miloso M, Cavaletti G and Tredici G: Role of MAPKs in platinum-induced neuronal apoptosis. Neurotoxicology 30: 312-319, 2009.

14. Owens DM and Keyse SM: Differential regulation of MAP kinase signaling by dual-specificity protein phosphatases. Oncogene 26: 3203-3213, 2007.

15. Katz M, Amit I and Yarden Y: Regulation of MAPKs by growth factors and receptor tyrosine kinases. Biochim Biophys Acta 1773: 1161-1176, 2007.

16. Montagut $C$ and Settleman J: Targeting the RAF-MEK-ERK pathway in cancer therapy. Cancer Lett 283: 125-134, 2009.

17. Garavello W, Nicolini G, Aguzzi A, Maggioni D, Leone BE, Viganò P, Gaini RM and Tredici G: Selective reduction of extracellular signal-regulated protein kinase (ERK) phosphorylation in squamous cell carcinoma of the larynx. Oncol Rep 16: 479-484, 2006.

18. Aguzzi A, Maggioni D, Nicolini G, Tredici G, Gaini RM and Garavello W: MAP kinase modulation in squamous cell carcinoma of the oral cavity. Anticancer Res 29: 303-308, 2009.

19. Munshi HG, Wu YI, Mukhopadhyay S, Ottaviano AJ, Sassano A, Koblinski JE, Platanias LC and Stack MS: Differential regulation of membrane type 1-matrix metalloproteinase activity by ERK 1/2- and p38 MAPK-modulated tissue inhibitor of metalloproteinases 2 expression controls transforming growth factor-beta1-induced pericellular collagenolysis. J Biol Chem 279: 39042-39050, 2004.

20. Werner JA, Rathcke IO and Mandic R: The role of matrix metalloproteinases in squamous cell carcinomas of the head and neck. Clin Exp Metastasis 19: 275-282, 2002.

21. Rosenthal EL and Matrisian LM: Matrix metalloproteases in head and neck cancer. Head Neck 28: 639-648, 2006. 
22. Yoshizaki T, Sato H and Furukawa M: Recent advances in the regulation of matrix metalloproteinase 2 activation: from basic research to clinical implication (Review). Oncol Rep 9: 607-611, 2002.

23. Bindhu OS, Ramadas K, Sebastian P and Pillai MR: High expression levels of nuclear factor kappa B and gelatinases in the tumorigenesis of oral squamous cell carcinoma. Head Neck 28: 916-925, 2006.

24. Kaomongkolgit R, Cheepsunthorn P, Pavasant P and Sanchavanakit N: Iron increases MMP-9 expression through activation of AP-1 via ERK/Akt pathway in human head and neck squamous carcinoma cells. Oral Oncol 44: 587-594, 2008.

25. Hammani K, Blakis A, Morsette D, Bowcock AM, Schmutte C, Henriet $\mathrm{P}$ and DeClerck YA: Structure and characterization of the human tissue inhibitor of metalloproteinases-2 gene. J Biol Chem 271: 25498-25505, 1996.
26. Raman M, Chen W and Cobb MH: Differential regulation and properties of MAPKs. Oncogene 26: 3100-3112, 2007.

27. Santen RJ, Song RX, McPherson R, Kumar R, Adam L, Jeng MH and Yue W: The role of mitogen-activated protein (MAP) kinase in breast cancer. J Steroid Biochem Mol Biol 80: 239-256, 2002.

28. Lee SH, Lee JW, Soung YH, Kim SY, Nam SW, Park WS, Kim SH, Yoo NJ and Lee JY: Colorectal tumors frequently express phosphorylated mitogen-activated protein kinase. APMIS 112: 233-238, 2004. 